\title{
THE ASYMPTOTIC BEHAVIOR OF A VOLTERRA-RENEWAL EQUATION(1)
}

BY

\author{
PETER NEY
}

Abstract. Theorem. Assume that the functions $x(\cdot), h(\cdot), G(\cdot)$ satisfy:

(i) $0 \leqslant x(t), t \in[0, \infty) ; x(t) \rightarrow 0$ as $t \rightarrow \infty ; x$ bounded, measurable;

(ii) $0 \leqslant h(s) ; h(s)$ Lipschitz continuous for $s \in I$, where $I$ is a closed interval containing the range of $x ; h(0)=0, h^{\prime}(0+)=1, h^{\prime \prime}(0+)<0$;

(iii) $G$ a probability distribution on $(0, \infty)$ having nontrivial absolutely continuous component and finite second moment.

Let $H x(t)=\int_{0}^{t} h[x(t-y)] d G(y)$. If $0 \leqslant(x-H x)(t)=o\left(t^{-2}\right)$, with strict inequality on the left on a set of positive measure, then $x(t) \sim \gamma / t, t \rightarrow \infty$, where $\gamma$ is a constant depending only on $h$ and $G$.

The condition $o\left(t^{-2}\right)$ is close to best possible, and cannot, e.g., be replaced by $O\left(t^{-2}\right)$.

1. Introduction and results. Let $\xi(\cdot)$ and $h(\cdot)$ be given nonnegative functions, $G$ a distribution function on $(0, \infty)$, and define the averaging operator

$$
H x(t)=\int_{0}^{t} h(x(t-y)) d G(y) .
$$

The equation

$$
x=\xi+H x
$$

arises in the study of deterministic and stochastic growth models such as particle multiplication, epidemics, and economic (capital) growth. (See F. Brauer [2] for references to some of these applications.) Our own encounter with (1) was motivated by a branching stochastic process. There $1-h(1-s)$ is a probability generating function governing particle production, $G$ is the particle life length distribution, $\xi(t)=s[1-G(t)]$ with $0 \leqslant s \leqslant 1$; and then $x(t)=x(t ; s)$ becomes the generating function of the number of particles in the process at time $t$. (See Chapter IV of Athreya and Ney [1].)

Received by the editors July 3, 1975 and, in revised form, November 15, 1975.

AMS (MOS) subject classifications (1970). Primary 45D05; Secondary 60K05.

Key words and phrases. Nonlinear Volterra equations, nonlinear renewal theory, Tauberian theorems, population growth, branching processes

(1) This research was supported by NSF grant MPS75-07113. 
Previous studies have established a variety of conditions for the existence of bounded solutions and for their convergence to a limit (see e.g. J. Levin [5], S. O. Londen [6], Athreya and Ney [1]). If such a limit (say $L$ ) exists, it is necessarily a root of

$$
L=\xi(\infty)+h(L)
$$

In the applications, the proofs of several classical limit theorems rest crucially on determination of the rate of convergence of $x(t)$ to $L$. To that end we initiated study of these rates with J. Chover [3] for the simplest branching model leading to (1), using techniques which depended on special aspects of the branching process.

The purpose of this paper is to extend one of the important "rate" theorems to a more general setting. The result is most naturally stated in a "Tauberian" form, namely that if $(x-H x)(t) \rightarrow 0$ fast enough, then the exact asymptotic form of $x(t)$ is determined.

In the interest of simplicity we assume that $\xi(\infty)=0, L=0=h(0)$, and hence $x(\infty)=0$. Cases in which $L$ is some other root of (2) can be reduced to this one with a little manipulation. It is natural that the rate of convergence should depend on whether $h^{\prime}(0)=1$ or $<1$ (one cannot have $x(t) \rightarrow 0$ when $h^{\prime}(0)>1$; see [2]). We here consider the case $h^{\prime}(0)=1$, which corresponds to the "critical case" in the probabilistic applications.

THEOREM. Assume that the functions $x(\cdot), h(\cdot)$, and $G(\cdot)$ satisfy the following conditions:

(i) $x$ is a nonnegative, bounded, measurable function on $[0, \infty)$, tending to 0 at $\infty$;

(ii) $h$ is a nonnegative, Lipschitz continuous function on a closed interval $I$ containing the range of $x$, such that $\left(^{2}\right)$

$$
h(0)=0, \quad h^{\prime}(0+)=1, \quad h^{\prime \prime}(0+)<0 ;
$$

(iii) $G$ is a probability distribution $(3)$ on $(0, \infty)$ having a nontrivial absolutely continuous component, and such that

$$
\int_{0}^{\infty} t^{2} d G(t)<\infty
$$

If

$$
0 \leqslant(x-H x)(t)=o\left(t^{-2}\right)
$$

(2) By $h^{\prime}(0+)=1$ we mean that $h^{\prime}(s)$ exists and is continuous in some neighborhood $(0, \delta), \delta>0$, and $h^{\prime}(s) \rightarrow 1$ as $s>0$. Similarly for $h^{\prime \prime}(0+)$.

(3) A right continuous, nondecreasing function such that $G(0+)=0, G(+\infty)=1$. 
with strict inequality (on the left) on a set of positive measure, then

$$
x(t) \sim \gamma / t, \quad t \rightarrow \infty,
$$

where

$$
\gamma=-\frac{2 \mu}{h^{\prime \prime}(0)}, \quad \mu=\int_{0}^{\infty} t d G(t)
$$

REMARK. Letting

$$
\xi(t)=(x-H x)(t)
$$

translates (5) into (1), with the hypothesis that $\xi$ is bounded and satisfies

$$
0 \leqslant \xi(t)=o\left(t^{-2}\right)
$$

and

$$
0<\xi(t)
$$

on a set of positive measure. Observe that the constant $\gamma$ is independent of $\xi(t)$ (subject to the restrictions (9), (10)).

The hypotheses of the theorem are essentially the best possible. In (3), $h(0+)=0$ and $h^{\prime}(0+)=1$ are, of course, necessary. Brauer [2] has shown that if $h^{\prime \prime}(0+)>0$ then $x(t) \rightarrow 0$ cannot occur. If $h^{\prime \prime}(0+)=0$ then there is a different asymptotic behavior. In fact $h(t)=t$ leads to the classical (linear) renewal equation, with $x(t) \rightarrow$ constant.

Condition (4) is known to be necessary in the branching process applications [1].

The rate $o\left(t^{-2}\right)$ also cannot be weakened, as is shown by taking $G(t)$ $=1-e^{-t}, t \geqslant 0 ; h(s)=\frac{1}{2}\left(1+s^{2}\right), 0 \leqslant s \leqslant 1 ; \xi(t)=c t^{-2}$. Then $x(t)$ $\sim$ const $/ t$, but the constant depends on $c$. If $\xi(t)=t^{-\alpha}$ with $0<\alpha<2$, then even the rate is changed.

Proceeding along the lines developed for the branching process in [3], one may, under stronger differentiability and moment conditions on $h$ and $G$, refine the result of the theorem. For example, under very slightly more than the existence of $h^{\prime \prime \prime}(0+)$, and a fourth moment of $G$, one can show that

$$
x^{-1}(t)=\gamma^{-1} t+\gamma_{1} \log t+\gamma_{2}+O\left(t^{-\gamma_{3}}\right),
$$

where $\gamma$ is as before, and $\gamma_{1}, \gamma_{2}, \gamma_{3}$ are constants, $\gamma_{1}$ depending on $h^{\prime \prime}(0)$, $h^{\prime \prime \prime}(0)$, and $\mu$. We will not go into such refinements here.

2. Proof of Theorem. The idea is to prove the Theorem for the special $h$ function 


$$
h_{a}(s)=\left(s^{-1}+a\right)^{-1}, \quad s \geqslant 0
$$

(Lemma 4), and then to show by a comparison argument that the result can be extended to general $h$. Lemmas 1,2 , and 3 prove uniqueness and other preliminaries about the solution, and about the iterates

$$
x_{n+1}=\xi+H x_{n} .
$$

These iterates (with $x_{0}(t)$ to be specified) will be used extensively.

LEMMA 1. Equation (1) has at most one solution which is bounded on compact sets.

Proof. Suppose that $x$ and $\bar{x}$ are two solutions such that $0 \leqslant x(s) \leqslant B$ $<\infty$ and $0 \leqslant \bar{x}(s) \leqslant B$ for $0 \leqslant s \leqslant t$. By hypothesis (ii) of the Theorem, $\left|h\left(y_{1}\right)-h\left(y_{2}\right)\right| \leqslant M\left|y_{1}-y_{2}\right|$ for some $M<\infty$ and for $\left(y_{1}, y_{2}\right) \in[0, B]$. Hence

$$
|x(t)-\bar{x}(t)| \leqslant M|x-\bar{x}| * G \leqslant \cdots \leqslant 2 B M^{n} G^{* n}(t) \rightarrow 0 \text { as } n \rightarrow \infty
$$

(see e.g. Lemma (IV.4.1) of [1]). (* denotes a convolution.)

LEMMA 2. If $h$ is nondecreasing on $I, x(t)$ is a bounded solution of (1), $0 \leqslant x_{0}(t) \leqslant x(t)$, and $x_{0}(t) \leqslant x_{1}(t)$ for $t \geqslant 0$, then $x_{n}(t)>x(t)$ (monotonely) as $n \rightarrow \infty$, and uniformly on compact $t$-sets. If $0 \leqslant x_{1}(t) \leqslant x_{0}(t) \in I$ for $t \geqslant 0$, then $x_{n}(t) \searrow x(t)$.

Proof. If $0 \leqslant x_{0} \leqslant x$ and $x_{0} \leqslant x_{1}$ then by the monotonicity of $h$,

$$
x_{1}=\xi+h\left(x_{0}\right) * G \leqslant \xi+h(x) * G=x,
$$

and

$$
x_{1}=\xi+h\left(x_{0}\right) * G \leqslant \xi+h\left(x_{1}\right) * G=x_{2} .
$$

Proceeding by induction, $0 \leqslant x_{n} \leqslant x_{n+1} \leqslant x, n \geqslant 1$. Thus (if $M$ is the Lipschitz constant and $I=[0, B]$ )

$$
\left|x_{n+1}-x_{n}\right|(t) \leqslant M\left|x_{n}-x_{n+1}\right| * G(t) \leqslant 2 B M^{n} G^{* n}(t) .
$$

Since $\sum M^{n} G^{* n}(t)<\infty, x_{n}(t) \nearrow$ some $\bar{x}(t) \leqslant x(t)$ uniformly on compact sets; and hence taking limits in (12), $\bar{x}$ is a solution of (1). By Lemma $1 \bar{x}=x$. The decreasing part is handled similarly.

LEMMA 3. Let $0 \leqslant x(t)$ be a bounded solution of (1) with $\xi(t)$ satisfying (9) and (10). Given any $\tau<\infty$, there exist $T<\infty, \delta>0$, and a function $\hat{\xi}(t)$ satisfying (9) and (10), such that $\hat{x}(t)=x(t+T)$ satisfies 
(i) $\hat{x}(t)>0$ for all $t \geqslant 0$,

(ii) $\hat{x}(t) \geqslant \delta$ for $0 \leqslant t \leqslant \tau$,

(iii) $\hat{x}=\hat{\xi}+H \hat{x}$.

Proof. We will define (successively) $t_{1}, t_{2}, t_{3}$, and, letting $T_{i}=t_{1}+\cdots$ $+t_{i}, i=1,2,3$, the corresponding functions

$$
\xi^{(i)}(t)=\xi\left(t+T_{i}\right)+\int_{t}^{t+T_{i}} h\left(x\left(t+T_{i}-y\right)\right) d G(y) .
$$

Letting $x^{(i)}(t)=x\left(t+T_{i}\right), i=1,2,3$, we observe that

$$
x^{(i)}(t)=\xi^{(i)}(t)+\left(H x^{(i)}\right)(t) .
$$

From the hypotheses on $\xi, h$, and $G$, it is immediate that $\xi^{(i)}, i=1,2,3$, all satisfy (9) and (10). We will see that there is a suitable choice of $T_{3}$ so that $x^{(3)}(t)>0$, and $\geqslant \delta>0$ for $0 \leqslant t \leqslant \tau$, for $\tau>0$, and taking $T=T_{3}$ will imply the lemma.

Note first that due to (3),

$$
h(s)=s+\left(h^{\prime \prime}(0) / 2\right) s^{2}+o\left(s^{2}\right), \quad s \searrow 0 .
$$

Hence there exist $\beta>0, s_{1}>0$, such that $\beta s<h(s) \leqslant 1$ for $0<s \leqslant s_{1}$, and since by hypothesis $x(t) \rightarrow 0$, there is a $t_{1}<\infty$ so that $x^{(1)}(t)=x\left(t+t_{1}\right)$ $\leqslant s_{1}, t \geqslant 0$. Defining $\xi^{(1)}(t)$ by (13), we observe from (14) that (since $\left.x^{(1)}(t) \leqslant s_{1}\right)$

$$
x^{(1)}(t) \geqslant \xi^{(1)}(t)+\beta\left(x^{(1)} * G\right)(t) .
$$

Iterating this relation yields

$$
x^{(1)}(t) \geqslant \int_{0}^{t} \xi^{(1)}(t-y) u(y) d y,
$$

where $u(y)=\sum \beta^{n} g^{* n}(y), g(\cdot)$ being the density associated with the absolutely continuous component of $G$. Let $|g|=\int_{0}^{\infty} g(t) d t$ and $v(y)$ $=\sum|g|^{-n} g^{* n}(y)$, and note that $u(y)>0$ if and only if $v(y)>0$. But by the renewal theorem (see Chapter XI of [4]) $\int_{0}^{t} \xi^{(1)}(t-y) v(y) d y \rightarrow$ const $>0$ ass $t \rightarrow \infty$; hence $x^{(1)}\left(t+t_{2}\right) \equiv x^{(2)}(t)>0$ for suitable $t_{2}<\infty$, where $x^{(2)}$ satisfies (14).

Arguing as in (15), and again using standard renewal theory (see e.g. Chapter IV of [1]),

$$
x^{(2)}(t) \geqslant \int_{0}^{t} \xi^{(2)}(t-y) u(y) d y \geqslant c e^{-\alpha t} \int_{0}^{t-t^{\prime}} \xi^{(2)}(y) d y
$$

for suitable $\alpha, c, t^{\prime}<\infty$. 
Hence for any $\tau$, there exist $0<\delta, t_{3}<\infty$, such that $x^{(2)}(t) \geqslant \delta$ on $\left[t_{3}, t_{3}+\tau\right)$. Letting $x^{(3)}(t)=x^{(2)}\left(t+t_{3}\right)$ implies the lemma.

REMARK. We will prove that the conclusion (6) of the Theorem holds for the function $\hat{x}$ in Lemma 3, i.e. that $\hat{x}(t)=x(t+T) \sim \gamma / t$. This, of course, implies the same result for $x(t)$. The exact form of $\hat{\xi}$ is not important, the only relevant fact being that it satisfies (9) and (10). Thus we see from Lemma 3 that it is no loss of generality to prove the Theorem for positive functions $\hat{x}(t)$ satisfying the conditions of the Theorem plus the additional restriction that for some $\delta>0, \tau>0$,

$$
\begin{array}{ll}
\hat{x}(t) \geqslant \delta>0 & \text { for } 0 \leqslant t \leqslant \tau, \\
\hat{x}(t)>0 & \text { for all } t \geqslant 0 .
\end{array}
$$

We will simplify our notation by dropping the 's from $x$ and $\xi$.

LEMMA 4. Equation (1) with $h=h_{a}$ (given in (11)) has a unique solution, say $x(t ; a)$, which satisfies the conclusion of the Theorem. Namely $x(t, a) \sim \mu / a \cdot 1 / t$, $t \rightarrow \infty$.

Proof. Note that $h_{a}$ is monotone. The iterates $x_{n}$, with $x_{0}=\xi$ are all well defined. Since $x_{n} \geqslant \xi$, we see by Lemma 2 that $x_{n}$ converges to a solution. Since $h_{a}$ and $\xi$ are bounded, any solution is bounded; and uniqueness follows from Lemma 1. To proceed with the proof it is convenient to let

$$
z(t)=1 / x(t ; a)+a,
$$

thus changing (1) into

$$
\frac{1}{z(t)-a}=\xi(t)+\int_{0}^{t} \frac{d G(y)}{z(t-y)} .
$$

Since $x$ is bounded, $z(t)>a+\eta$ for some $\eta>0$.

We start by establishing bounds on $z(t)$. First we claim that

$$
\delta t \leqslant z(t) \text { for some } \delta>0 .
$$

To prove this fact start with the iterates $z_{n}(t)=x_{n}^{-1}(t ; a)+a$, with

$$
z_{0}(t)= \begin{cases}a & \text { for } t \leqslant t_{0} \\ \varepsilon t & \text { for } t>t_{0}\end{cases}
$$

where $0<t_{0}<\infty$ is arbitrary and fixed, and $\varepsilon>0$ is to be specified. Clearly $z_{1}(t) \geqslant a$ for $t \leqslant t_{0}$. Suppose $t>t_{0}$ and write 


$$
\begin{aligned}
\frac{1}{z_{1}(t)-a} & =\xi(t)+\int_{0}^{t-t_{0}} \frac{d G(y)}{z_{0}(t-y)}+\int_{t-t_{0}}^{t} \frac{d G(y)}{z_{0}(t-y)} \\
& \leqslant \xi(t)+\frac{1}{\varepsilon t}\left\{1+\int_{0}^{t-t_{0}} \frac{y d G(y)}{t-y}\right\}+\frac{1}{a}\left[1-G\left(t-t_{0}\right)\right],
\end{aligned}
$$

and note that (4) implies that $\int_{0}^{t-t_{0}} y d G(y) /(t-y)=O\left(t^{-1}\right)$. Combining the above terms, and using (4) and (9), some calculation yields

$$
1 /\left(z_{1}(t)-a\right) \leqslant\{1+K / t\} / \varepsilon t, \quad t \geqslant t_{0},
$$

for some $K<\infty$ depending on $t_{0}$. Hence $z_{1}(t) \geqslant \varepsilon t-\varepsilon K+a \geqslant \varepsilon t$, provided $0<\varepsilon<a / K$. By Lemma $2, z_{n}(t)>z(t)$, and this implies (19).

Turning to an upper bound, we show next that

$$
z(t)=O(t), \quad t \rightarrow \infty .
$$

From (19) and Lemma $3, z(t)$ is bounded above and away from 0 on bounded intervals. For $0<\tau<\infty$ we may thus let $z_{n}=\sup \{z(t):(n-1) \tau \leqslant t \leqslant n \tau\}$, and $\gamma_{n}$ $=G(n \tau)$. By (19) and hypothesis (iii) of the theorem it is possible to take $N$ sufficiently large and $\tau$ small, so that for some $\alpha<1$

$$
\left(\frac{1-\gamma_{1}}{\gamma_{n}-\gamma_{1}}\right)\left(1+\frac{\gamma_{1}}{1-\gamma_{1}} \frac{a}{z_{n+1}}\right) \leqslant 1+\frac{\alpha}{n} \text { for } n \geqslant N .
$$

Take $C$ so that $z_{j} \leqslant C j$ for $j=1, \ldots, N$, and $C>a /(1-\eta)$. We will prove by induction that $z_{n} \leqslant C n$, thus implying (20). Suppose that $z_{k} \leqslant C k$ for $k \leqslant n$, where $n \geqslant N$. Taking the inf of both sides of (18) over $t \in[n \tau$, $(n+1) \tau]$ yields

$$
\frac{1}{z_{n+1}-a} \geqslant \inf \sum_{j=0}^{\infty} \int_{j \tau}^{(j+1) \tau} \frac{d G(y)}{z(t-y)} \geqslant \frac{\gamma_{1}}{z_{n} \vee z_{n+1}}+\sum_{j=1}^{n-1} \frac{\gamma_{j+1}-\gamma_{j}}{z_{n-j} \vee z_{n-j+1}} .
$$

If $z_{n+1} \leqslant z_{n}$ we are finished. If $z_{n+1}>z_{n}$ the above inequalities, together with the induction hypothesis and (21) imply that

$$
\frac{1}{z_{n+1}-a}-\frac{\gamma_{1}}{z_{n+1}} \geqslant \sum_{j=1}^{n} \frac{\gamma_{j+1}-\gamma_{j}}{(n-j+1) C} \geqslant \frac{\gamma_{n}-\gamma_{1}}{C n},
$$

and thus $z_{n+1}-a \leqslant C n(1+\alpha / n)$, and $z_{n+1} \leqslant C(n+1)$. (If $\gamma_{1}=0$ the above argument must be slightly modified, but is even easier.)

Continuing with the proof of Lemma 4, rewrite (18) as

$$
z^{-1}(t)=r(t)+z^{-1}(t) * G(t)
$$

where 


$$
r(t)=\xi(t)-a z^{-1}(t)(z(t)-a)^{-1} .
$$

Though the forcing term depends on the unknown $z(\cdot)$, we know enough about $z$ from (19) and (20) to apply the renewal theorem. From a form of the latter due to C. Stone [6], we obtain from (23) that

$$
z^{-1}(t)=\int_{0}^{t} r(t-y) d V(y)+\int_{0}^{t} r(t-y) w(y) d y,
$$

where $\int_{0}^{\infty} y^{2} d V(y)<\infty$, and $w(t)=\mu^{-1}+\rho(t)$, with $\rho=o\left(t^{-1}\right), \int_{0}^{\infty} \rho(t) d t$ $<\infty$. By hypothesis and (19), $r(t)=O\left(t^{-2}\right)$, and hence (25) implies that

$$
z^{-1}(t)=o\left(t^{-1}\right)+\mu^{-1} \int_{0}^{t} r(y) d y .
$$

Since $z^{-1}(t) \rightarrow 0$ as $t \rightarrow \infty$ we must have that $\int_{0}^{\infty} r(y) d y=0$, or

$$
z^{-1}(t)=o\left(t^{-1}\right)-\mu^{-1} \int_{t}^{\infty} r(y) d y .
$$

Now from (24) we get

$$
\int_{t}^{\infty} r(y) d y=\int_{t}^{\infty} \xi(y) d y-a^{2} \int_{t}^{\infty} \frac{d y}{z^{2}(y)[z(y)-a]}-a \int_{t}^{\infty} \frac{d y}{z^{2}(y)} .
$$

The estimates (9) and (19) imply that the first two terms on the right-hand side are $o\left(t^{-1}\right)$, and hence

$$
z^{-1}(t)=\gamma(t)+\frac{a}{\mu} \int_{t}^{\infty} z^{-2}(y) d y
$$

where $\gamma(t)=o\left(t^{-1}\right)$.

Finally, letting $\zeta(t)=\int_{t}^{\infty} z^{-2}(y) d y,(26)$ can be rewritten as

$$
(d / d t)(1 / \zeta(t))=(\gamma(t) / \zeta(t)+a / \mu)^{2} .
$$

Since (due to (20)) $\zeta(t) \geqslant$ const $/ t$, the right side of (27) tends to $a / \mu$ as $t \rightarrow \infty$. Hence $1 / \zeta(t) \sim(a / \mu)^{2} t$ as $t \rightarrow \infty$. Resubstituting this in (26) implies Lemma 4.

To complete the proof of the Theorem let $x(t)$ be a solution of (1) such that $x(t) \rightarrow 0$, and let $a=-h^{\prime \prime}(0+) / 2$. From the Taylor expansion for $h$ and $h_{a}$ we see that given any $\varepsilon>0$ there is an $s_{0}$ such that $h_{a+\varepsilon}(s) \leqslant h(s) \leqslant h_{a-\varepsilon}(s)$ for $0 \leqslant s \leqslant s_{0}$, and by (3) we may also take $s_{0}$ small enough so that $h(\cdot)$ is monotone increasing for $0 \leqslant s \leqslant s_{0}$. Next choose a $t_{0}$ such that $x(t)$ $\leqslant s_{0}$ for $t \geqslant t_{0}$. Letting $\hat{x}(t)=x\left(t+t_{0}\right)$, rewrite (1) as

$$
\hat{x}(t)=\hat{\xi}(t)+\int_{0}^{t} h(\hat{x}(t-y)) d G(y)
$$


where

$$
\hat{\xi}(t)=\xi\left(t+t_{0}\right)+\int_{t}^{t+t_{0}} h\left(x\left(t+t_{0}-y\right)\right) d G(y) .
$$

Let $x^{+}(t)$ be the solution of $x^{+}=\hat{\xi}+h_{a-\varepsilon}\left(x^{+}\right) * G$. Let

$$
\hat{x}_{0}(t)=x_{0}^{+}(t)=\hat{\xi}(t) \leqslant \hat{x}(t)=x\left(t_{0}+t\right) \leqslant s_{0} .
$$

Then

$$
\hat{x}_{1}=\hat{\xi}+h(\hat{\xi}) * G \leqslant \hat{\xi}+h_{a-\varepsilon}(\hat{\xi}) * G=x_{1}^{+} .
$$

Suppose $\hat{x}_{n} \leqslant x_{n}^{+}$. (Note $\hat{x}_{n}(t) \leqslant \hat{x}(t) \leqslant s_{0}$ since $h$ is monotone on the range of $\hat{x}$.) Then

$$
\hat{x}_{n+1}=\hat{\xi}+h\left(\hat{x}_{n}\right) \leqslant \hat{\xi}+h_{a-\varepsilon}\left(\hat{x}_{n}\right) * G \leqslant \hat{\xi}+h_{a-\varepsilon}\left(x_{n}^{+}\right) * G=x_{n+1}^{+},
$$

and thus by induction $\hat{x}(t) \leqslant x^{+}(t)$. Letting $x^{-}$be the solution of $x^{-}$ $=\hat{\xi}+h_{a+\varepsilon}\left(x^{-1}\right) * G$, we show similariy that $x^{-}(t) \leqslant \hat{x}(t)$. Thus

$$
x^{-}(t) \leqslant x\left(t+t_{0}\right) \leqslant x^{+}(t), \quad t \geqslant 0
$$

and by Lemma 4

(28) $\lim _{t \rightarrow \infty} t x^{-}(t)=\frac{\mu}{a+\varepsilon} \leqslant \lim _{t \rightarrow \infty} t x\left(t+t_{0}\right) \leqslant \lim _{t \rightarrow \infty} t x^{+}(t)=\frac{\mu}{a-\varepsilon}$.

Since $\varepsilon$ is arbitrary this proves the Theorem.

\section{REFERENCES}

1. K. Athreya and P. Ney, Branching processes, Springer-Verlag, Berlin and New York, 1972.

2. F. Brauer, On a nonlinear integral equation for population growth problems, SIAM J. Math. Anal. 6 (1975), 312-317.

3. J. Chover and P. Ney, The non-linear renewal equation, J. Analyse Math. 21 (1968), 381-413. MR 39 \# 7690.

4. W. Feller, An introduction to probability theory and its applications. Vol. II, 2nd ed., Wiley, New York, 1971.

5. J. Levin, On a nonlinear Volterra equation, J. Math. Anal. Appl. 39 (1972), 458-476. MR 46 \#4124.

6. S.-O. Londen, On a nonlinear Volterra integral equation, J. Differential Equations 14 (1973), 106-120. MR 49 \# 5745.

7. C. Stone, On absolutely continuous components and renewal theory, Ann. Math. Statist. 37 (1966), 271-275. MR 33 \#4981.

Department of Mathematics, University of Wisconsin-Madison, Madison, Wisconsin 53706 